\title{
Finite element modeling of the lost foam casting process tackling back-pressure effects
}

\author{
Guillaume Houzeaux and Ramon Codina \\ International Center for Numerical Methods in Engineering, Barcelona, Spain
}

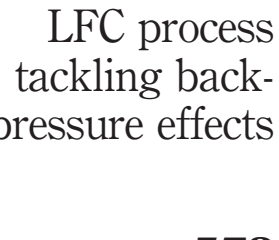

573

Received January 2005

Revised September 2005

Accepted November 2005

\begin{abstract}
Purpose - To develop a numerical methodology to simulate the lost foam casting (LFC), including the gas back-pressure effects.

Design/methodology/approach - Back-pressure effects are due to the interactions of many physical processes. The strategy proposed herein tries to model all these processes within a simple formula. The main characteristic of the model consists of assuming that the back-pressure is a known function of the external parameters (coating, temperature, gravity, etc.) that affects directly the heat transfer coefficient from the metal to the foam. The general framework of the simulation is a finite element model based on an arbitrary Lagrangian Eulerian (ALE) approach and the use of level set function to capture the metal front advance.

Findings - After experimental tunings, the model provides a way to include the back-pressure effects in a simple way.

Research limitations/implications - The method is not completely predictive in the sense that a priori tuning is necessary to calibrate the model.

Practical implications - Provides more realistic results than classical models.

Originality/value - The paper proposes a theoretical framework of a finite element method for the simulation of LFC process. The method uses an ALE method on a fixed mesh and a level-set function to capture metal front advance. It proposes an original formula for the heat transfer coefficient that enables one to include back-pressure effects.
\end{abstract}

Keywords Foaming agents, Gas flow, Simulation, Finite element analysis

Paper type Research paper

\section{Introduction}

In this paper we present a numerical model for the simulation of a relatively new casting process, known as lost foam casting (LFC) or evaporative pattern casting. See Piwonka (1990) for an introduction to LFC. This process was developed during the 1960s and has known an exponential growth since then. To have an idea of the importance gained by this technology in the last years, let us just mention that around 20 per cent of the total aluminum casting in the USA is obtained by LFC (Divone, 1999). Despite its important place on the casting market, numerical codes have only recently allowed the numerical simulation of LFC. This is partly due to the complexity of the physical processes in play.

The authors want to thank Martin Solina from Quantech ATZ for providing us with the gravity casting simulation results. This work was carried out in the framework of the European Project FOAMCAST G1RD-CT-2000-00417.

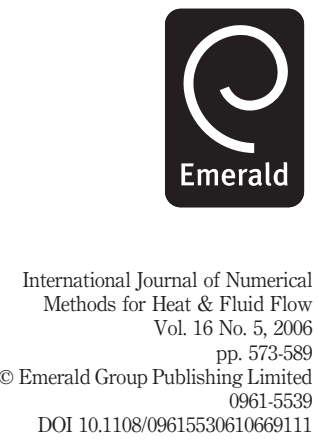


$\mathrm{HFF}$

16,5

\section{4}

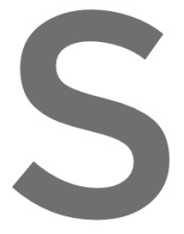

Register for free at $h^{\text {t }}$
In general casting, known as gravity casting, the molten metal is poured into a mould filled with air. The metal is allowed to fill the mould thanks to gravity, if the pressure at the inflow is sufficiently high. In LFC, the air is replaced by expandable polystyrene (EPS). The polystyrene bead is placed in the mould, it is heated and expands until it completely fills the mould. The resulting cluster is dipped into a permeable refractory coating (of alumina, silica), which will act as a barrier between the sand and the metal, providing a control on the gases escape and a better surface finish. It is then embedded in a container filled with compacted sand and provided with a gating. LFC operates as follows. The molten metal is poured into the gating and once it gets in contact with the foam, the foam evaporates: this process is known as pyrolysis. Gases can vent through the sand if the coating is sufficiently permeable. However, if the gas is not vented sufficiently fast, casting defects can appear and perturb the metal advance. The main parameters that control the process are the EPS pattern and combustion characteristics, the coating material, thickness, texture and permeability.

The numerical approach to LFC requires special attention at the physical and numerical level. Owing to the complex physical mechanisms involved, some physical simplifications are needed. For example, we decide not to try solving for the combustion of the foam. Instead, we obtain an equation for the metal front velocity although velocity generatio 1998). T involves characteristic velocities
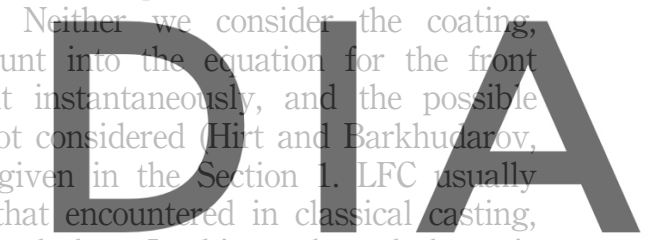

and the metal flow can be either laminar or turbulent. In this work, turbulence is

alternative consists in prescribing the pressure at front. This approach has the

advantage that it naturaily takes into account the back-pressure effects due to the decomposition of the EPS pattern. A possible implementation is explained in Kuo et al. (2003); this method, however, does not consider the heat transfer rate between metal and foam as an explicit part of the front advance mechanism. See also Mirbagheri et al. (2003), where the authors propose in addition a model for the back-pressure.

The numerical approximation chosen to solve the present problem is the finite element method. In order to capture the ever changing computational domain occupied by the metal, three main approaches are available (Lewis and Ravindran, 2000). The first method consists in writing the governing equations in a Lagrangian frame of reference, the nodes being advected by the flow velocity (Muttin et al., 1993; Radovitzky and Ortiz, 1998). The second method is based on an Eulerian formulation. The position of the front is identified by a level set function (LSF) (Osher and Fedkiw, 2002), which is transported by the velocity (Codina et al., 1994; Chang and Yang, 2001). The third method is the arbitrary Lagrangian Eulerian (ALE) method, which uses both Lagrangian and Eulerian approaches, and consists in adapting the mesh to each new configuration but in limited zones; generally this implies a node movement near the front and a remeshing if the resulting mesh is too distorted (Lewis et al., 1997; Gaston et al., 2000). Let us mention finally the meshless method (Idelsohn et al., 2001) to solve 
free surface flows using a Lagrangian formulation. In this work we have chosen an ALE method, but with the originality that it is applied to a fixed mesh (FMALE).

This paper is a continuation of the work initiated in (Houzeaux and Codina, 2004), where some of the modelling and numerical aspects presented here are described in more detail. Apart from summarizing the material of this reference, we concentrate here in the modelling of the back-pressure effects. We introduce them through a particular law for the heat transfer coefficient between the metal and the foam that depends on the flow pressure and the gas pressure generated from the foam combustion. This law incorporates also temperature effects by multiplying the gas pressure by a function of temperature that we adjust from the experimental results of Yao and Shivkumar (1995). Likewise, we also present a new industrial application that demonstrates the capability of the present model to simulate LFC.

\section{Physical model}

\subsection{Simplifying assumption}

We described in the Section 1 the complexity of the physical processes involved in LFC. In order to treat LFC numerically, some simplifying assumptions are needed. In addition, assumptions are added for the sake of clarity of the exposition. They are:

- Metal and foam properties are constant.

S
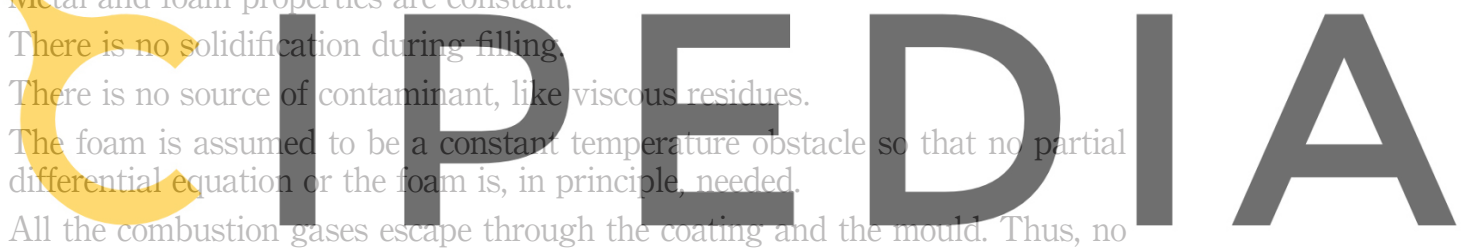

gas is trapped between the metal and the foam.

Register for free at https//www.scipedia.com to download the version without the watermark According to these simplifications, we are able to devise a concise physical model, presented in the following.

\subsection{Governing equations}

Let $\Omega_{\mathrm{m}}$ the domain that is filled by the molten metal, $\Omega_{\mathrm{f}}$ the domain occupied by the foam and $\Omega$ the total domain (metal + foam). They are shown in Figure 1. Obviously, both domains depend on time.

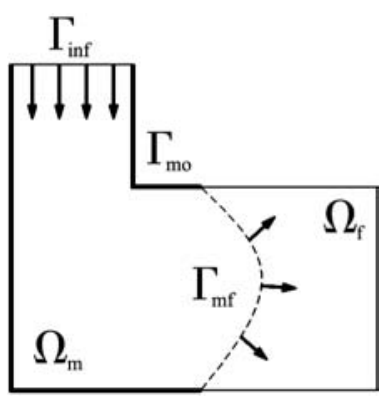

Figure 1. Domain and boundary notation 
$\mathrm{HFF}$

16,5

576

Let us denote the physical properties as follows: $\rho$ as the density, $\nu$ as the kinematic viscosity, $c_{\mathrm{p}}$ as the specific heat at constant pressure, $\kappa$ as the thermal diffusion coefficient and $\alpha_{i j}$ as the heat transfer coefficient between materials $i$ and $j$. The unknowns to be determined in the general case are $\mathbf{u}$ the velocity, $p$ the pressure and $T$ the temperature of the molten metal. Let us denote $u_{\mathrm{mf}}$ as the velocity at which the front of molten metal advances through the foam.

The subscripts $\mathrm{m}, \mathrm{f}$ and o will be used to refer to molten metal, foam and mould, respectively, for the physical properties. Likewise, $\Gamma_{i j}$ will be used to denote the interface between materials $i$ and $j$, and subscript inf will refer to values at the inflow of the domain (Figure 1).

The equations describing the lost foam model in an Eulerian frame of reference are conservation of momentum, mass and energy, given by:

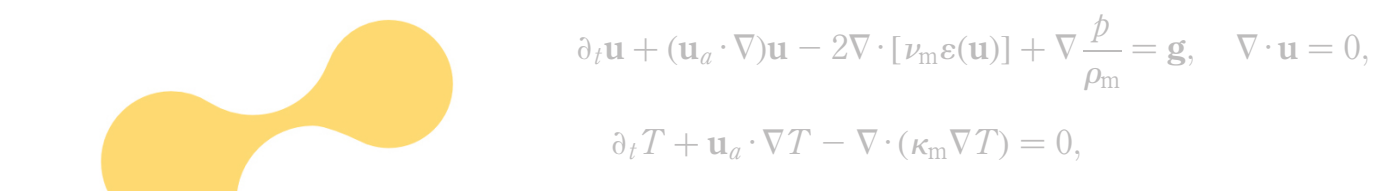

to be solved in $\Omega_{\mathrm{m}}$, where $\varepsilon(\mathbf{u})$ is the rate of deformation tensor given by:
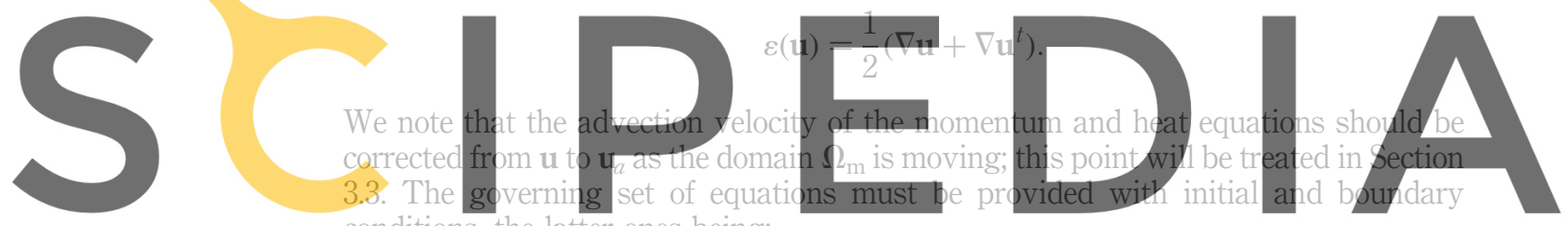

conditions, the latter ones being:

Register for free at https//www.scipedia.com to download the versipm without the watermark

$$
\begin{array}{cc}
\mathbf{u}=0 & \text { on } \Gamma_{\mathrm{mo}}, \\
\mathbf{u}=\mathbf{u}_{\mathrm{mf}} & \text { on } \Gamma_{\mathrm{mf}}, \\
T=T_{\mathrm{inf}} & \text { on } \Gamma_{\mathrm{inf}}, \\
-\kappa_{\mathrm{m}} \nabla T \cdot \mathbf{n}=\alpha_{\mathrm{mo}}\left(T-T_{\mathrm{o}}\right) /\left(\rho_{\mathrm{m}} c_{p \mathrm{~m}}\right) & \text { on } \Gamma_{\mathrm{mo}}, \\
-\kappa_{\mathrm{m}} \nabla T \cdot \mathbf{n}=\alpha_{\mathrm{mf}}\left(T-T_{\mathrm{f}}\right) /\left(\rho_{\mathrm{m}} c_{p \mathrm{~m}}\right) & \text { on } \Gamma_{\mathrm{mf}} .
\end{array}
$$

These conditions are, respectively, a traction boundary condition, the no-slip condition on the walls, the front advance velocity condition, the prescription of the pouring temperature, the prescription of the heat flux on the walls and the prescription of the heat flux between metal and foam. The vector $\mathbf{n}$ is the outward unit normal to domain $\Omega_{\mathrm{m}}$, the tensor $\boldsymbol{\sigma}$ is the stress tensor given by:

$$
\boldsymbol{\sigma}=-p I+2 \rho_{\mathrm{m}} \nu_{\mathrm{m}} \varepsilon(\mathbf{u})
$$

$I$ being the $n_{\mathrm{d}}$-dimensional identity. $T_{\text {inf }}$ is the pouring temperature and $p_{\text {inf }}$ is the pouring pressure. We note that the boundary condition given by equation $(2)_{1}$ at the inflow is: 


$$
p=p_{\text {inf }} \quad \text { on } \Gamma_{\text {inf }},
$$

only if $\varepsilon(\mathbf{u})=0$, which is satisfied, for example, if the flow is uniform.

In the governing set of equations, apart from the velocity, pressure and temperature, we are left with two unknowns, namely the front advance velocity and the time dependent domain $\Omega_{\mathrm{m}}$. A model for the front advance velocity is now given. Next a front advance tracking is presented to tackle the evolution of $\Omega_{\mathrm{m}}$.

Remark. Equation (2) 2 can be replaced by a slip condition, that is by imposing a zero tangential traction together with a zero normal velocity.

\subsection{Front velocity}

The front velocity at which the front advances is a function of the heat absorbed by the foam, which in turn depends on u and $T$ so that $u_{\mathrm{mf}}=u_{\mathrm{mf}}(\mathbf{u}, T)$. If it is high enough, the foam will be molten and vaporized, and the room left free will be occupied by the metal. The front velocity can be computed from a simple global balance of energy. Suppose that a point of the interface molten metal-foam advances a distance $\delta$ s normal to the surface of the interface in a time $\delta t$. The heat per unit surface released from the molten metal to the volume initially occupied by foam will be:

\section{LFC process tackling back- pressure effects}

577

\section{$Q_{\mathrm{rel}}=\alpha_{\mathrm{mf}}\left(T_{\mathrm{m}}-T_{\mathrm{f}}\right) \delta \mathrm{s}$
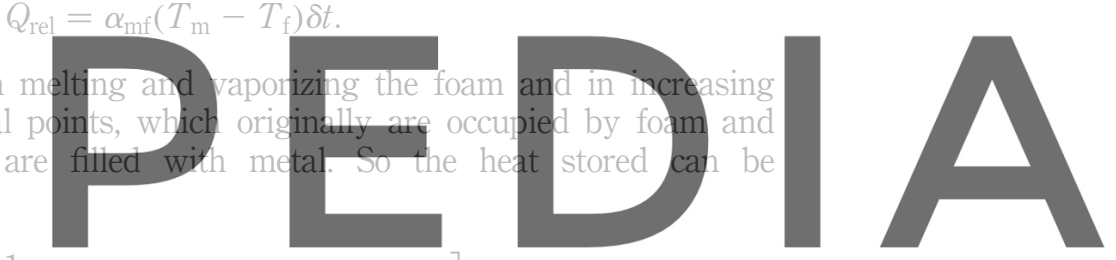 \\ Register for free at https//Www.scipedia.com to download the version without the watermark}

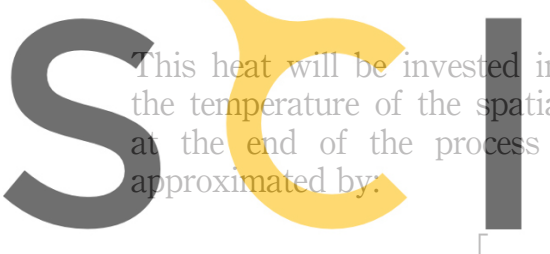

where $E_{\text {mel }}$ and $E_{\mathrm{vap}}$ are the melting and vaporization energy, respectively, which must be determined from experiments. Imposing that $Q_{\text {sto }}=Q_{\text {rel }}$ it is found that:

$$
\rho_{\mathrm{f}} c_{p \mathrm{f}} \frac{\delta s}{\delta t}\left(T_{\mathrm{m}}-T_{\mathrm{f}}\right)+\frac{\delta s}{\delta t}\left(\rho_{\mathrm{f}} E_{\mathrm{mel}}+\rho_{\mathrm{f}} E_{\mathrm{vap}}\right)=\alpha_{\mathrm{mf}}\left(T_{\mathrm{m}}-T_{\mathrm{f}}\right)
$$

For $\delta t \rightarrow 0, \delta s / \delta t \rightarrow u_{\mathrm{mf}}$, from where:

$$
u_{\mathrm{mf}}=\frac{\alpha_{\mathrm{mf}}\left(T_{\mathrm{m}}-T_{\mathrm{f}}\right)}{\rho_{\mathrm{f}}\left(c_{p \mathrm{f}}\left(T_{\mathrm{m}}-T_{\mathrm{f}}\right)+E_{\mathrm{mel}}+E_{\mathrm{vap}}\right)} .
$$

We note that the model for the front velocity does not take into account the effects of pouring temperature, coating, foam type, gravity, etc. In fact, the front velocity is independent on the mould orientation with respect to the gravity. From experiments, the front advance should depend on the pouring temperature, the coating, the foam and the gravity $\mathbf{g}$ (Liu et al., 1994; Wang et al., 1994; Yao and Shivkumar, 1995). In addition, more drastic effects can occur. For high temperature and low coating permeabilities, a large gap between the front and the burnt foam is observed, called the kinetic zone (Bakhtiyarov and Overleft, 2003). This situation is not envisaged in the context of this work. 
$\mathrm{HFF}$

16,5

\section{8}

is a known function.

We now present an expression for the metal-to-foam heat transfer coefficient expliciting its dependence on $p_{\text {gas }}$. We assume that the heat transfer coefficient in the absence of back-pressure and gravity effects is known, and is referred to as $\alpha_{\mathrm{mf}}^{0}$. It can be shown that the value of the heat transfer between two materials is proportional to their separation distance $d$. If we assume next that this distance is proportional to the pressure difference between the metal and the gas, then we can express the heat transfer as a function of these pressures. In particular, we have:

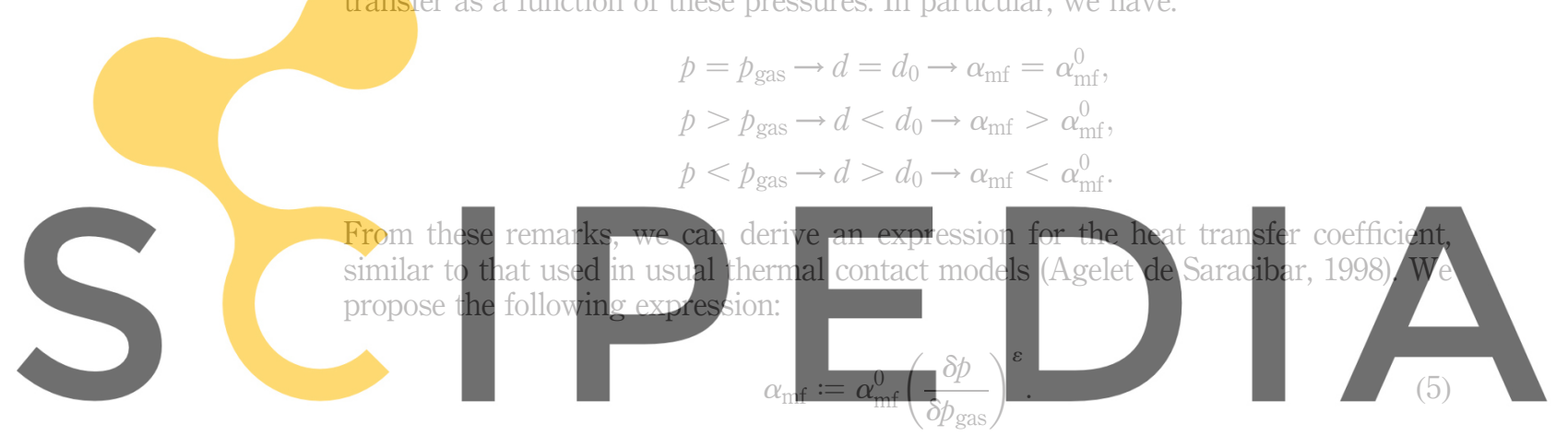

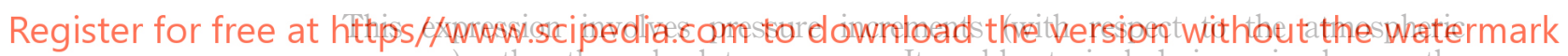
pressure) rather than absolute pressures. It enables to include in a simple way the effects of the set of parameters ( $T_{\mathrm{inf}}$, foam, coating, g) in the determination of the front advance, as the front velocity $u_{\mathrm{mf}}$ depends explicitly on the heat transfer coefficient. Indeed, we have assumed that we knew an expression for the gas pressure in function of the pouring temperature, the foam and the coating, given by equation (4). The dependence on the gravity appears implicitly through the dependence on the pressure in equation (5). We have then:

$$
u_{\mathrm{mf}}=\frac{\alpha_{\mathrm{mf}}\left(T_{\mathrm{inf}}, \text { foam, coating, } \mathbf{g}\right)\left(T_{\mathrm{m}}-T_{\mathrm{f}}\right)}{\rho_{\mathrm{f}}\left[c_{p \mathrm{f}}\left(T_{\mathrm{m}}-T_{\mathrm{f}}\right)+E_{\mathrm{mel}}+E_{\mathrm{vap}}\right]} .
$$

\subsection{Front tracking}

One possibility of defining the molten metal-foam interface (or equivalently to determine $\Omega_{\mathrm{m}}$ at each time) is the level set technique. This is not a general conservation law, but rather a mathematical model to describe the evolution of this interface (see Codina et al. (1994) for an application of this method to classical mould fillings). It consists in defining a scalar function, called the LSF, say $\psi$, over the computational domain $\Omega$ in such a manner that its value at a certain point indicates the presence or absence of metal. This function may be considered a fictitious fluid property. For instance, we may assign the value 1 to regions where the liquid metal has already 
entered and the value 0 to foam-filled regions. The position of the metal front will be defined by the isovalue contour $\psi=\psi_{c}$ where $\psi_{c} \in[0,1]$ is a critical value defined a priori. We usually take $\psi_{c}=0.5$. This value is immaterial if $\psi$ is a true step function, but is needed in the finite element discretization. The level set technique is usually used to follow free surfaces of fluids, i.e. surfaces advected by the fluid velocity itself. In the present case we have a somewhat different situation. The LSF is advected due to temperature gradients and is, therefore, not explicitly advected by the fluid motion (equation (3)).

The velocity at the metal front is assumed to move along the normal to the front, which is precisely the gradient of the level set function:

$$
\mathbf{u}_{\mathrm{mf}}=-\frac{\nabla \psi}{|\nabla \psi|} u_{\mathrm{mf}}
$$

\section{Numerical model \\ In the previous section we presented the physical modeling of the lost foam process. However, some purely numerical ingredients are necessary to solve the governing equations efficiently. We first introduce the finite element method which is used to solve the partial differential equations. Next we introduce the treatment of the front

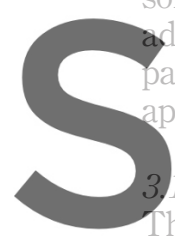 \\ dvance. Then we explai \\ articular we will see tha plied to fixed meshes. \\ Finite element method and time discretization \\ he Navier-Stokes and heat equation
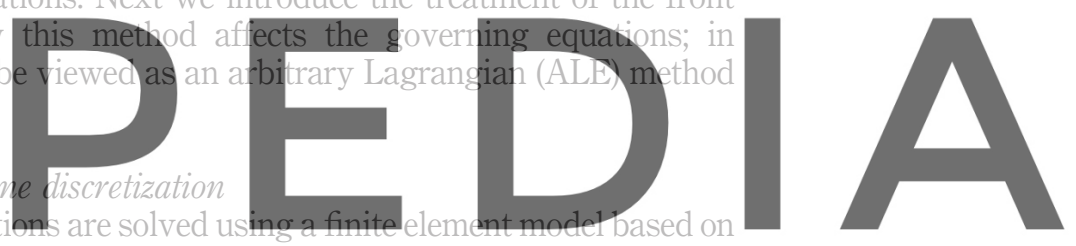

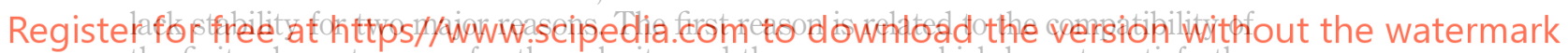 \\ the finite element spaces for the velocity and the pressure which have to satisfy the}

so-called Ladyzhenskaya-Brezzi-Babuška condition. This condition is necessary to obtain a stability estimate for the pressure; without requiring this condition, the pressure would be out of control. The second reason is attributed to the relative importance of the viscous and convective effects in the momentum equation. The stabilized formulation is based on the algebraic variational SGS model first introduced in Hughes (1995). The variational SGS model uses as a starting argument that the inability of the mesh to resolve all the flow scales is responsible for the numerical instabilities. Therefore, the model calculates in some approximate way the unresolved scales of the flow, i.e. the scales smaller than the mesh size. The method is extensively described in Codina (2001). Finally, the time discretization is carried out using to generalized trapezoidal rule, i.e. a finite difference scheme. To do so, we consider a partition $0=t^{0}<t^{1}<\cdots<t^{N}=T$ of the time interval $[0, T]$ of interest. The time step is denoted as $\delta t=t^{n}-t^{n-1}$.

In this work, we consider two types of elements using both equal order interpolation for the velocity and the pressure. The $Q 1 / Q 1$ element is continuous and bilinear (trilinear in three dimensions) in both velocity and pressure. We will also work with the $P 1 / P 1$ element, continuous and linear in velocity and pressure.

A similar numerical model is used to solve the equation for the temperature $T$, and the LSF $\psi$, which are interpolated like $\mathbf{u}$ and $p$. They are integrated in time using 
$\mathrm{HFF}$

16,5

\section{0}

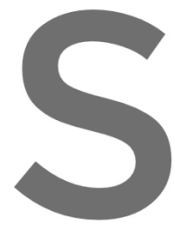

the generalized trapezoidal rule and the algebraic SGS method is employed to stabilize the possible dominance of convective term. See Codina (2001) for further details.

\subsection{Front advance treatment}

In Section 2.4, we mentioned the possibility of identifying the presence and absence of metal by solving a partial differential equation for the LSF. In this work, we propose to solve the partial differential equations in the whole domain $\Omega$ in order to advect the LSF correctly across the interface. We thus have to define in a clear way the metal domain, the domain of interest. As presented here, the proposed method is exclusive to the finite element method (although it can surely be extended to other methods).

Let us take as reference the solution obtained at a time $t^{n}$. We assume that the LSF $\psi^{n}$ is known. The idea is to divide the finite element mesh of $\Omega$ into three zones, which are three sets of elements of the finite element discretization: the metal set with elements whose nodes satisfy $\psi>\psi_{c}$; the foam set with elements whose nodes satisfy $\psi \leq \psi_{c}$; and finally, the front set which includes elements that have both metal and foam nodes, i.e. the remaining elements. In the same way, we define the front nodes as the nodes belonging to front elements, which in addition can be of metal or foam. Metal nodes are nodes of metal elements; likewise, we define foam nodes similarly. These metal and foam nodes can be of front type also.
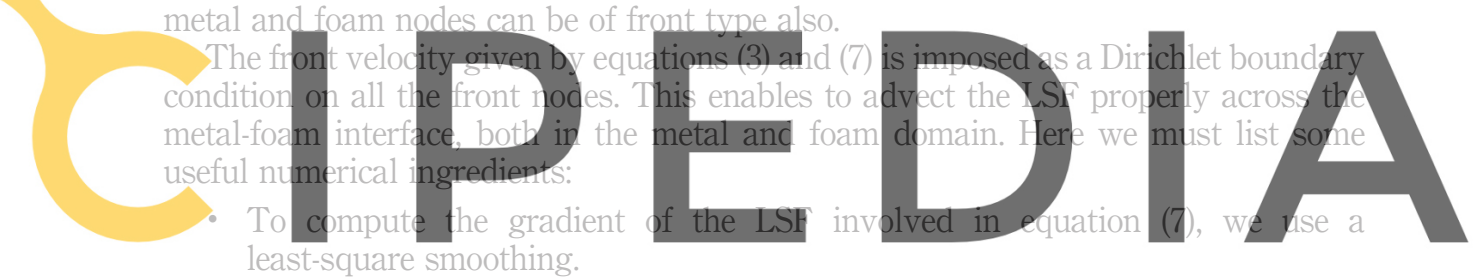

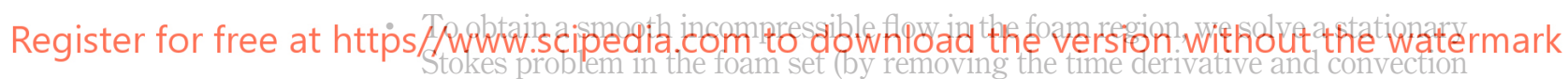
term). In addition, all the boundary nodes of the foam set are left free so that the artificial fluid in the foam can escape the domain and be incompressible. That is, we impose:

$$
\sigma \cdot \mathbf{n}=0 \quad \text { on } \Gamma_{\text {fo }} .
$$

This step can be understood as an extension of the velocity field in the metal region to foam. It is necessary for the numerical problem (not for the continuous one) since at each time step the front will enter several foam nodes where the velocity needs to be defined.

- To insulate the metal set completely from the foam set, the element matrices of the front set are not assembled in the global stiffness matrix of the Navier-Stokes and heat equations. Note that for numerical reasons, it is preferable to multiply the front element matrices by a small value (say $10^{-6}$ ) rather than removing them from the assembly. What we have now are two independent domains, one filled with metal, the other filled with foam, and a front of one element width on which the velocity is prescribed to $\mathbf{u}_{\mathrm{mf}}$.

- The velocity vectors of the front nodes located on the mould boundary are projected on the boundary so that the metal does not escape the metal domain. 
- As far as the heat equation is concerned, by removing the front element contribution to the stiffness matrix we are imposing implicitly an adiabatic condition for the metal domain, that is $\alpha_{\mathrm{mf}}=0$ in equation (2) $)_{6}$. How ever, if $\alpha_{\mathrm{mf}} \neq 0$, the adiabatic condition can be converted into the desired boundary condition by reconstructing the boundary. In the independent foam domain, the temperature can be set constant.

\subsection{ALE method with fixed mesh}

When using the level set technique in general casting, involving a metal-air mixture, the Navier-Stokes equations are solved in the entire mould, the physical properties being those of the metal or those of the air according to the value of $\psi$. The frame of reference is then Eulerian. In the present case, the LSF is used to define the metal computational domain, which changes at all time. However, according to the numerical algorithm used to capture the front advance, we want to use the same mesh all along the time integration. The method to solve this problem is an ALE method with a fixed mesh. The convection velocity $\mathbf{u}_{a}$ in equations $(1)_{1-3}$ should be equal to $\mathbf{u}-\mathbf{u}_{\mathrm{m}}$ where $\mathbf{u}_{\mathrm{m}}$ is the mesh velocity of the new nodes of the metal domain and $\mathbf{u}$ elsewhere. The method is described in Houzeaux and Codina (2004).

\section{Numerical examples}

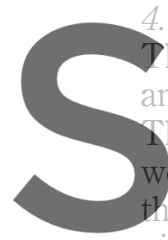

1.1 Horizontal plate

The first example we sol and Shivkumar, 1995; Hir

The values of the physica

set $\alpha_{\mathrm{mo}}=0$ in equation

is example we want to
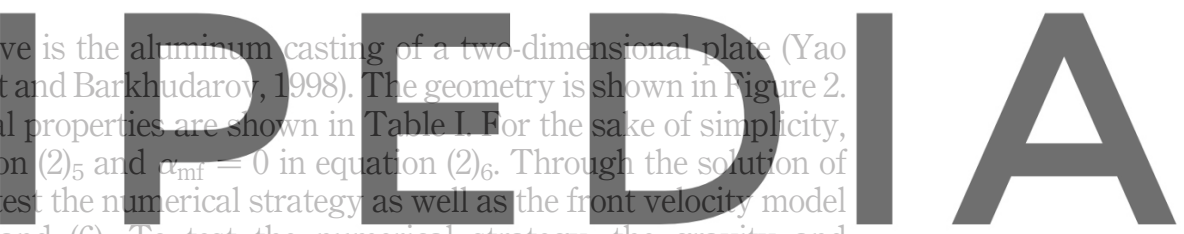

given by equations (5) and (6). To test the numerical strategy, the gravity and

back-pressure effects are neglected by putting $\varepsilon=0$ in equation.(5). Then we consider

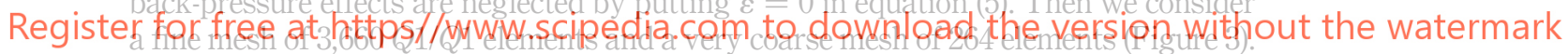

On the fine mesh, both the no-slip and a slip condition for the velocity are put to the

test, as well as a large time step $\delta t=0.1 \mathrm{~s}$ and a small one $\delta t=0.05 \mathrm{~s}$.

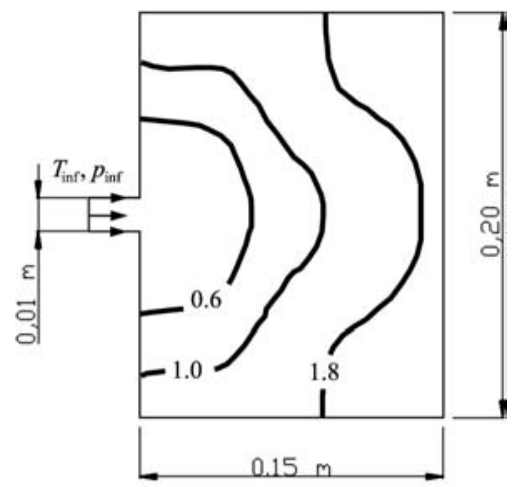

Notes: Geometry, boundary conditions and experimental front advance for $\mathrm{T}$ $=988 \mathrm{~K}$ and side gating

Figure 2.

Source: Yao and Shivkumar (1995) Horizontal plate 


\section{$\mathrm{HFF}$ 16,5

Symbol Property Value \\ Aluminum \\ $\alpha_{\mathrm{mf}} \quad$ Metal to foam heat transfer coefficient \\ Density \\ $582 \quad \begin{array}{ll}\nu_{\mathrm{m}} & \text { Viscosity } \\ \kappa_{\mathrm{m}} & \text { Conductivity }\end{array}$ \\ Specific heat \\ Inflow temperature \\ Inflow pressure \\ $6.00 \times 10^{3}\left(\mathrm{~kg} \mathrm{~s}^{-3} \mathrm{~K}^{-1}\right)$ \\ $2.70 \times 10^{3}\left(\mathrm{~kg} \mathrm{~m}^{-3}\right)$ \\ $3.70 \times 10^{-7}\left(\mathrm{~m}^{2} \mathrm{~s}^{-1}\right)$ \\ $8.32 \times 10^{-5}\left(\mathrm{~m}^{2} \mathrm{~s}^{-1}\right)$ \\ $c_{p \mathrm{~m}}$ \\ $p_{\text {inf }}$ \\ $8.37 \times 10^{2}\left(\mathrm{~J} \mathrm{~kg}^{-1} \mathrm{~K}^{-1}\right)$ \\ $9.88 \times 10^{2}(\mathrm{~K})$ \\ $4.00 \times 10^{3}(\mathrm{~Pa})$

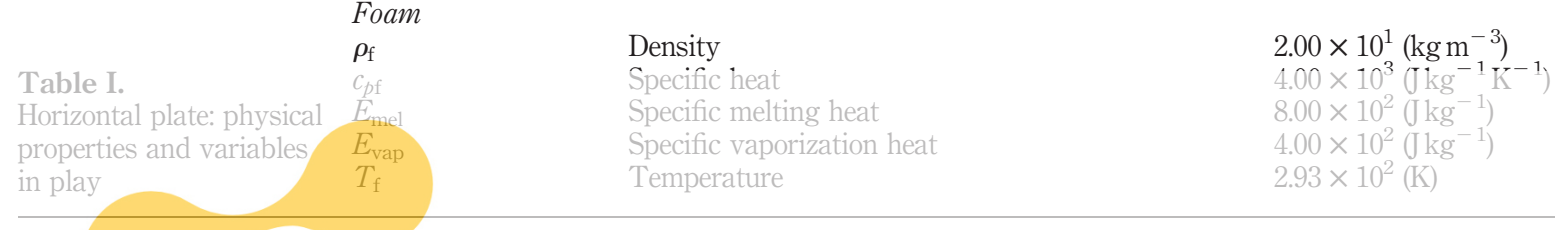
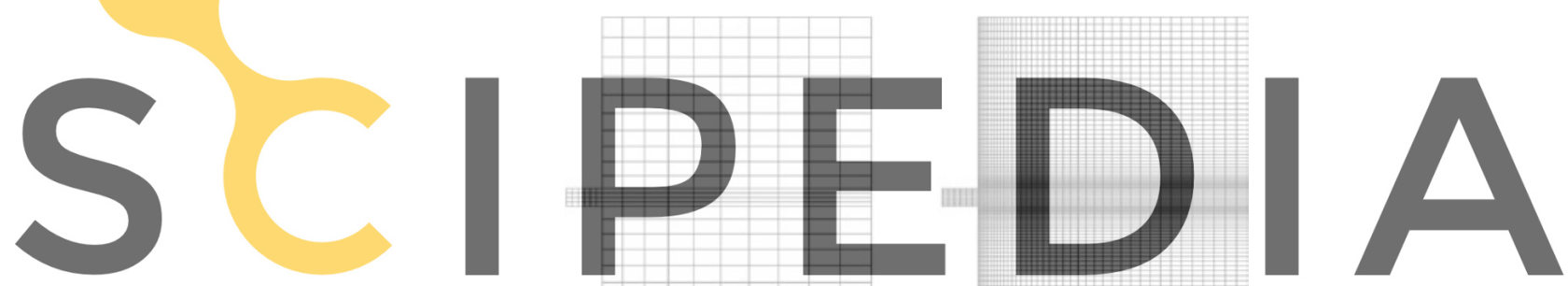 \\ Register for free at https//www.scipedia.com to download the version without the watermark

Figures 4 and 5 compare the evolutions of the metal front and velocity vectors for the three times 0.6, 1.0 and $1.8 \mathrm{~s}$ shown in Figure 2. For the sake of comparisons, the time is set to zero when the front passes the plate entrance. We first observe that the distortioned profile of the front obtained in the experiments cannot be well reproduced. Note also that taking $\varepsilon=0$ the front velocity is independent of the inflow temperature as we have:

$$
u_{\mathrm{mf}} \approx \frac{\alpha_{\mathrm{mf}}}{\rho_{\mathrm{f}} c_{p \mathrm{f}}} .
$$

Then, contrary to the experiment, we would obtain the same front evolution for any inflow temperature.

We also observe a strong recirculation zone generated once the metal enters the plate, and transported by the metal jet from left to right. This recirculation is even captured by the (very) coarse mesh, but located at a different position. We also observe that using the no-slip condition, higher velocities are obtained in the jet. This is a mesh size effect as in order for the no-slip solution to pump the same flow rate from the ingate as the slip solution, the velocity of the no-slip simulation must be greater at 

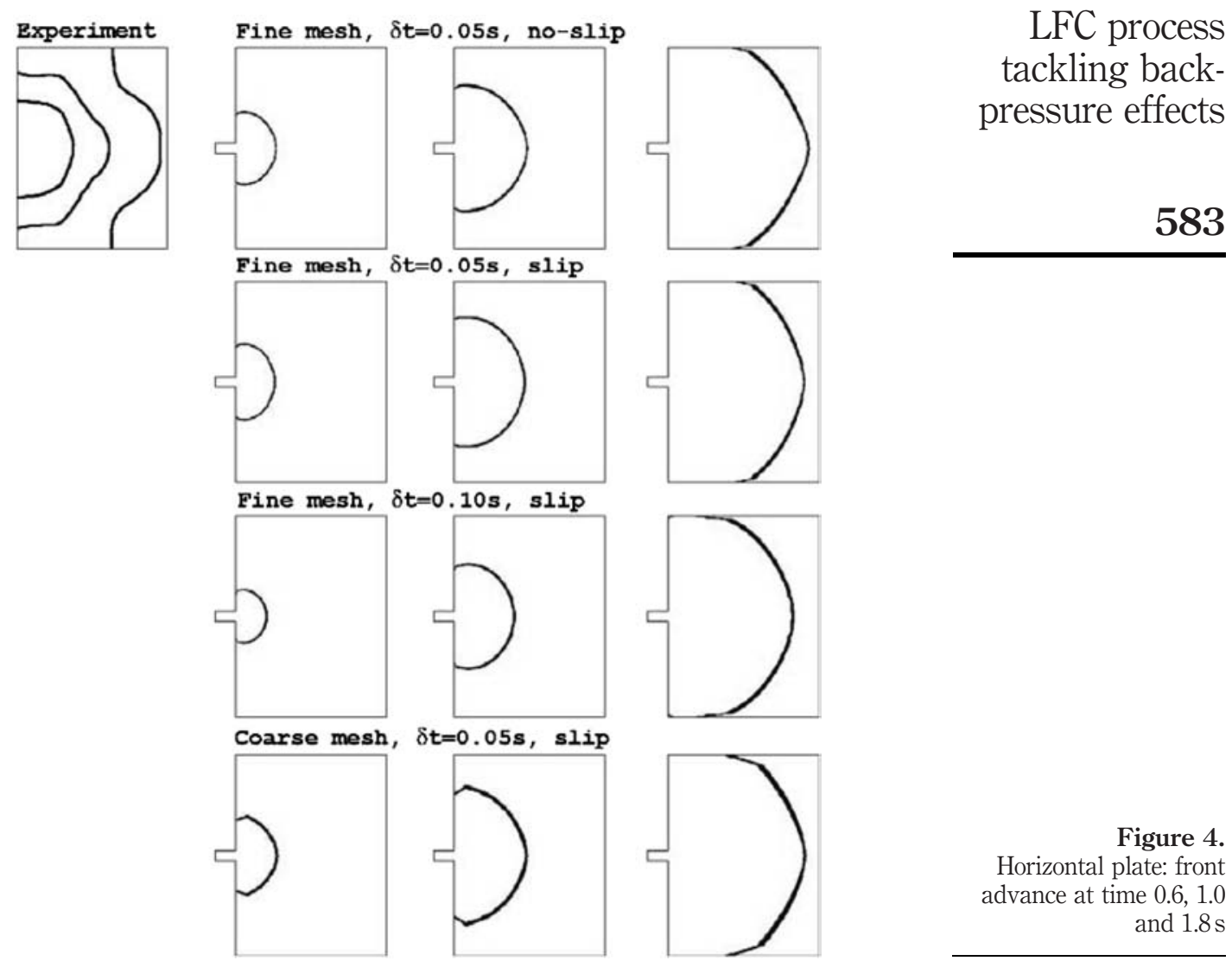

583

the ingate. The generation of this vortex is shown in more detail in Figure 6. This figure also shows the temperature contours.

Figure 7 compares the velocity vectors obtained in the top left part of the plate using the slip and no-slip conditions. We note that the profiles are similar.

We now test the model for the front advance with gas back-pressures. For this example we take $\varepsilon=1$ in equation (5) and set:

$$
\alpha_{\mathrm{mf}}=\alpha_{\mathrm{mf}}^{0} \frac{p+\rho_{\mathrm{m}} g h}{p_{\text {gas }} \beta(T)},
$$

where $p_{\text {gas }}=4 \times 10^{3} \mathrm{~Pa}$. This value belongs to the range of experimental gas pressures given in Liu et al. (2002). It is equal to $p_{\text {inf }}$ given from Table I where $p_{\text {inf }}$ can be, for example, provided by a downsprue. $h$ is the distance in the gravity direction, in this case from the ingate. $g=9.8 \mathrm{~m} \mathrm{~s}^{-2}$ is the norm of the gravity vector. The function $\beta(T)$ represents the temperature dependence of the back-pressure. It is chosen in order to reproduce approximately the experimental results of Yao and Shivkumar (1995). We set:

$$
\beta(T)=\left(-5.33 \times 10^{-4} T_{\text {inf }}+1.4\right)^{-1},
$$




\section{HFF \\ 16,5}

584

Figure 5.

Horizontal plate: velocity vectors at time $0.6,1.0$ and $1.8 \mathrm{~s}$

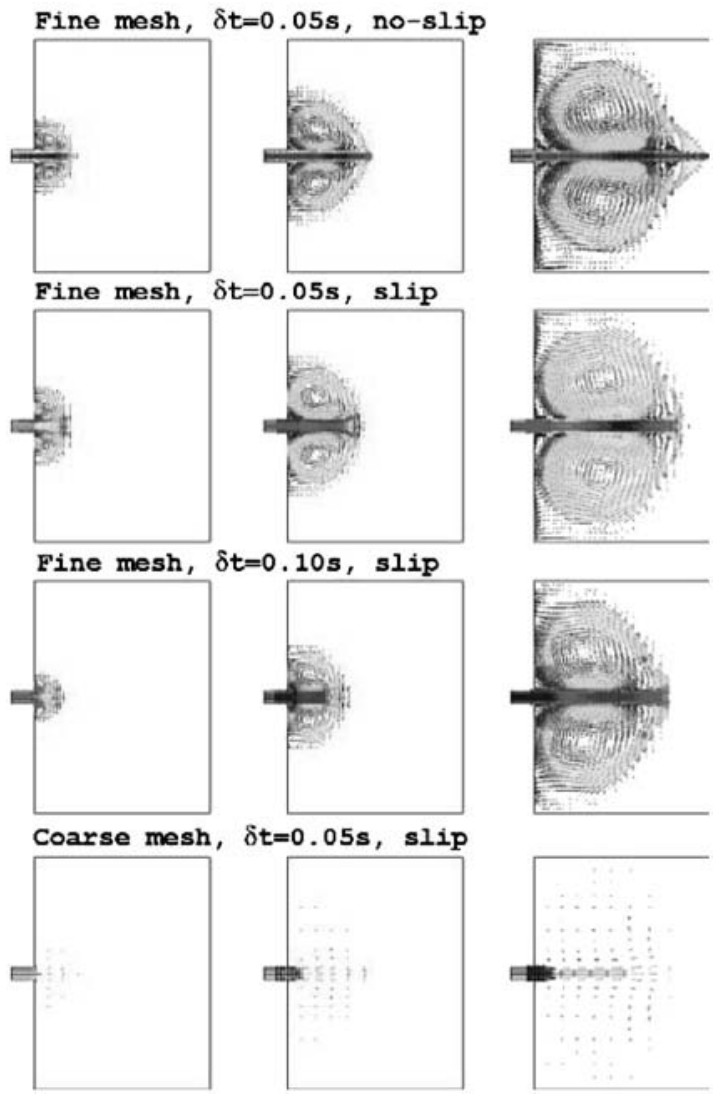

Figure 6.

Horizontal plate: metal entering the plate; velocity vectors (top); temperature contours (bottom)
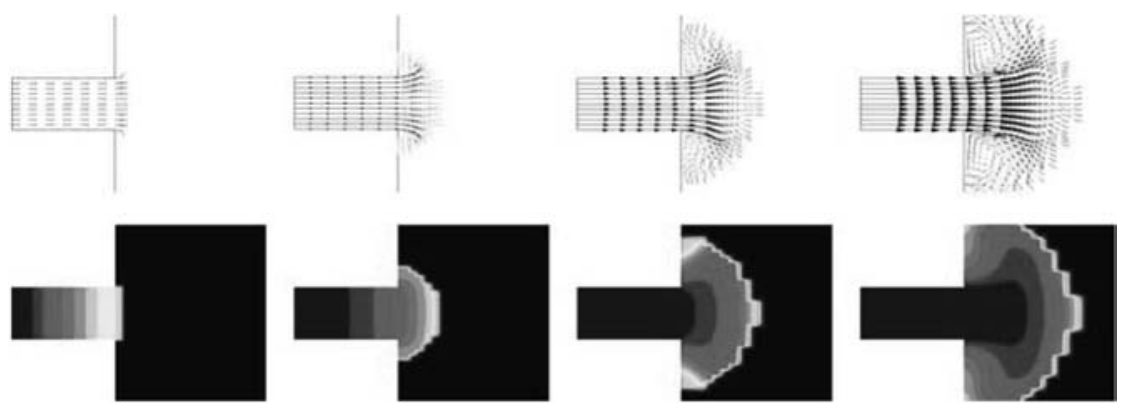
which is valid for $T \geq 763 \mathrm{~K}$. Note that $\beta(T=763)=1$ so that the heat transfer (and thus the velocity) is maximum, as indicated by the experimental data. Observe also that this expression implies that when $T$ increases then $\beta(T)$ increases and thus the front velocity $u_{\mathrm{mf}}$ decreases, whereas one would expect a faster burning of the front and, therefore, a higher front velocity. In general terms, the physical effects that could explain this behaviour are the impermeability of the coating (which could prevent gases from escaping and, therefore, increase the back pressure) or the generation of residues in the front where combustion takes place. In any case, the expression for $\beta(T)$ given is the one that adjusts sufficiently well the experimental data for the temperature range considered.

Table II shows the filling times obtained for three different inflow temperatures $T_{\text {inf }}=763,988,1,388 \mathrm{~K}$ without the gravity term in equation (8); this situation would correspond to a side gating configuration of the experiment. These filling times are also compared to the one obtained for $T_{\mathrm{inf}}=988 \mathrm{~K}$ with a top gating, that is taking into account the gravity term in equation (8).

Finally, Figure 8 compares the profiles obtained at different times of the four cases presented before. We observe that with respect to the results obtained with $\alpha_{\mathrm{mf}}=\alpha_{\mathrm{mf}}^{0}$ as shown in Figure 4, the front advance is faster in the centre region. This increase in the front velocity is a consequence of the high mechanical pressure encountered in this region, whose dependence is taken into account explicitly in the heat transfer coefficient model (equation (8)).

\subsection{Revolver lathe piece}

We now simulate a three-dimensional casting and compare the results obtained with a lostfoam casting with that of a gravity casting. The geometry is given in Figure 9 (Left), and the mesh, composed of 160,785 tetrahedral elements, is shown in Figure 9 (Right). This is a real geometry of a real casting example but for which we do not have
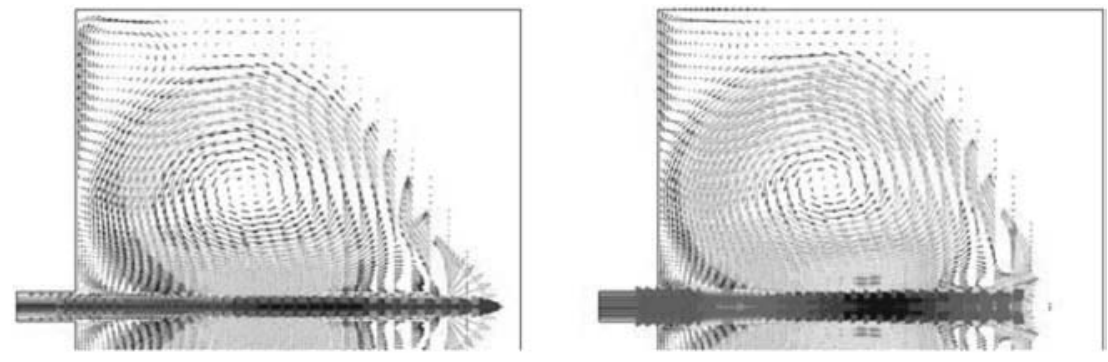

Figure 7. Horizontal plate: velocity vectors at top left corner, fine mesh, $\delta t=0.05 \mathrm{~s}$; no-slip condition on walls

(left); slip condition on walls (right)

\section{LFC process tackling back- pressure effects}

585

\begin{tabular}{llcc}
\hline$T_{\text {inf }}(\mathrm{K})$ & Gating & Time $(\mathrm{s})$ & Experiment (s) \\
\hline 988 & Top & 1.65 & 1.75 \\
763 & Side & 2.25 & 2.10 \\
988 & Side & 2.50 & 2.70 \\
1,388 & Side & 3.30 & 3.15
\end{tabular}

Source: Yao and Shivkumar (1995)
Table II.

Horizontal plate: filling time for different temperatures and gating compared with experimental results 


\section{$\mathrm{HFF}$ \\ 16,5}

\section{6}
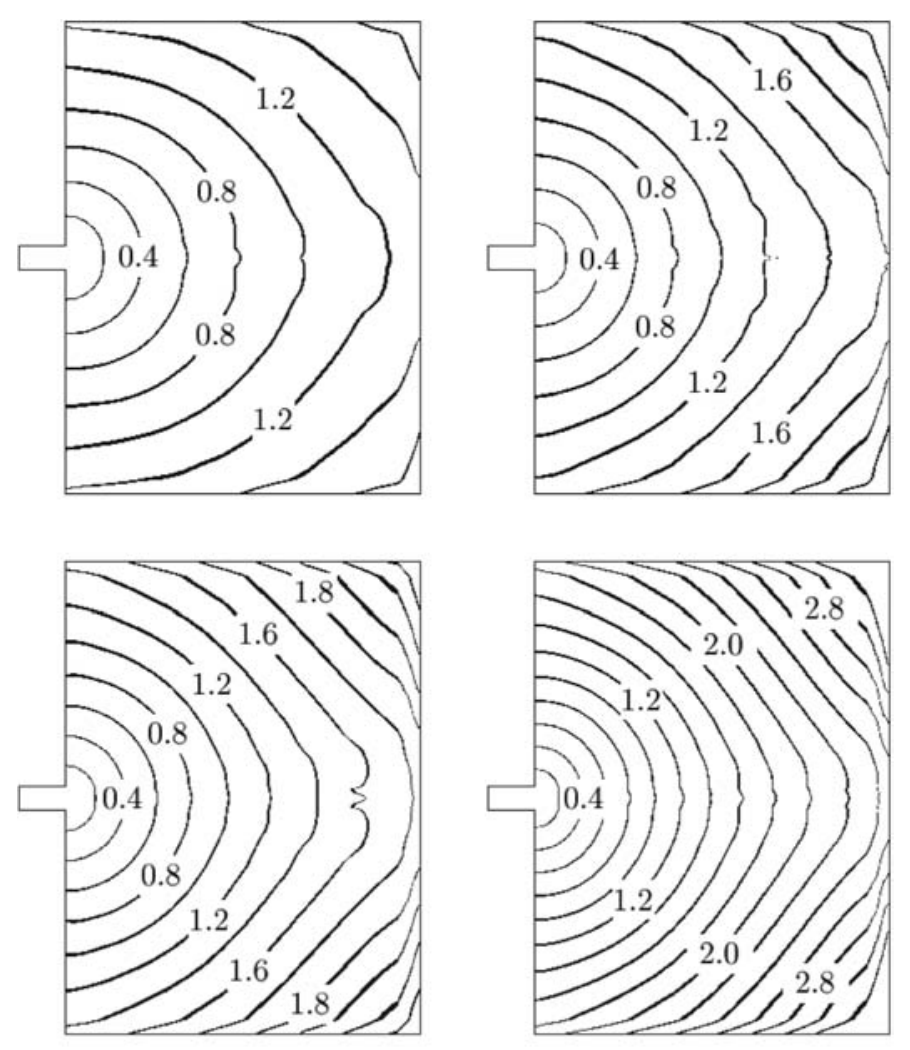

Figure 8.

Horizontal plate
Notes: (Top) (Left) Top gating, $T_{\text {inf }}=988 \mathrm{~K}$. (Top) (Right) Side gating, $T_{\text {inf }}=763 \mathrm{~K}$. (Bot.) $\left(\right.$ Left) Side gating, $T_{\text {inf }}=988 \mathrm{~K}$. (Bot.) (Right) Side gating, $T_{\text {inf }}=1388 \mathrm{~K}$

experimental results to compare with. This example is merely intented to understand qualitatively the difference between classical gravity casting and LFC. We take the same physical properties as those of the previous example, and for the front velocity model, we take $\varepsilon=0$ in equation (5). Figure 10 shows the evolution of the metal front for both castings. The red colour is used to depict the interior metal front while the grey colour indicates the metal-mould contact surface. On the one hand, we observe that in the gravity casting, the ingate cone is filled at last. On the other hand, in the case of the lostfoam casting, the metal advance is more isotropic; in fact, the model for the front advance chosen here does not depend on gravity, as $\varepsilon=0$.

Finally, Figure 11 shows the velocity vectors and the temperature contours obtained with the lostfoam simulation at two different times of the casting.

\section{Conclusions}

We have presented a numerical model to solve LFC problems. This numerical model is based on a finite element method using an ALE formulation with a fixed mesh. It was found that the evolution of the metal domain could be well captured by solving 

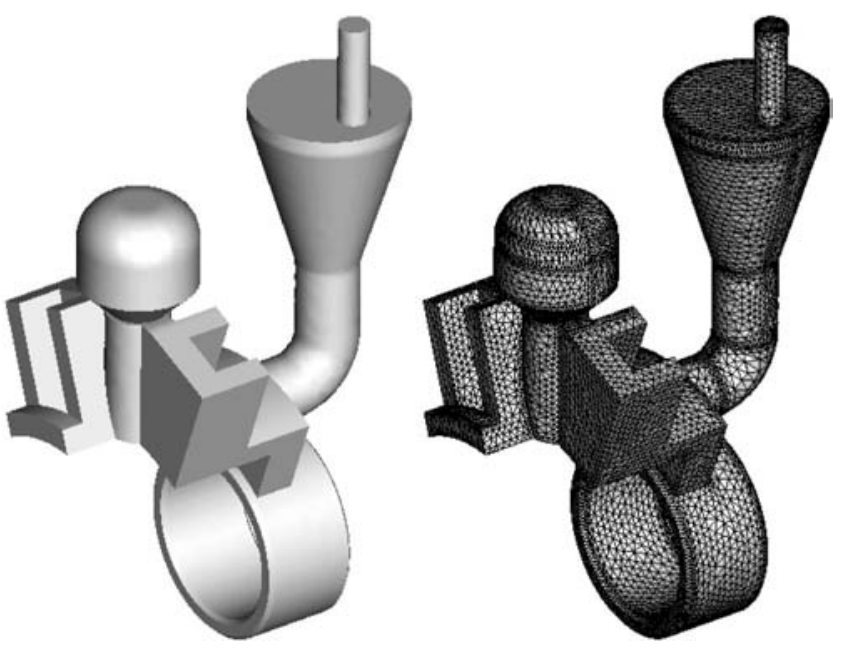

\section{LFC process tackling back- pressure effects}
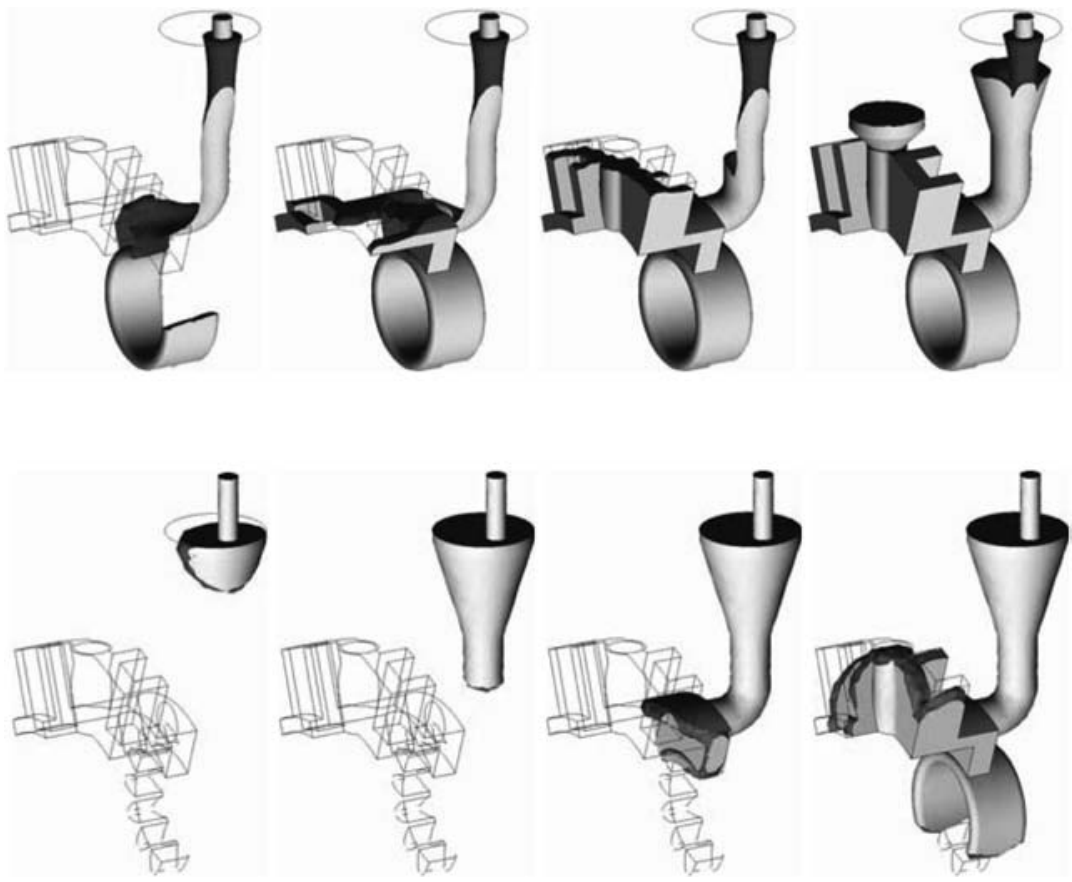

Figure 10.

Revolver lathe piece: metal advance at different time steps; gravity casting (top); lostfoam casting (bottom)

a partial differential equation for a level set function, advected on both sides of the front. The first numerical example showed that the dynamics of the metal was almost exclusively driven by the front advance, as the different time steps, the different wall treatment and the meshes give similar results. 


\section{HFF \\ 16,5}

\section{8}

Figure 11.

Revolver lathe piece: velocity vectors and temperature contours
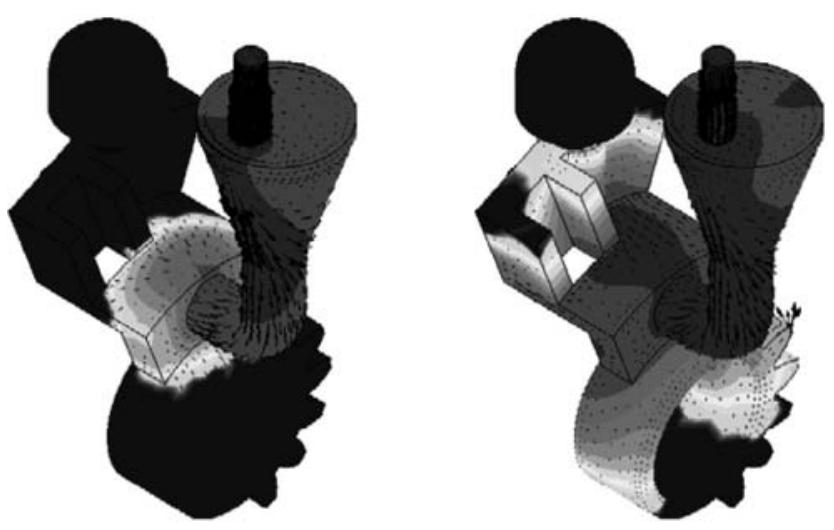

A model for the front velocity has been devised. It has been calibrated using experimental data on a simple geometry. Such a calibration can then be useful for solving complex three-dimensional castings, as long as all the important physical parameters are kept constant (foam, coating thickness, etc.).

\section{References}

Agelet de Saracibar, C. (1998), "Numerical analysis of coupled thermomechanical frictional contact problems. Computational model and applications", Arch. Comp. Meth. Eng., Vol. 5 No. 3, pp. 243-301.

Bakhtiyarov, S.I. and Overleft, R.A. (2003), "Cfd simulation of fluid flow through porous media: application to decomposed gases flow through foam pattern in lost foam casting", Proceedings of IMECE'03, 2003, ASME International Mechanical Engineering Congress, Number IMECE2003-43500, Washington, DC, November.

Chang, R.Y. and Yang, W.H. (2001), "Numerical simulation of mould filling in injection moulding using a three-dimensional finite volume approach", Int. J. Num. Meth. Fluids, Vol. 37 No. 2, pp. $125-248$.

Codina, R. (2001), "A stabilized finite element method for generalized stationary incompressible flows”, Comp. Meth. Appl. Mech. Eng., Vol. 190, pp. 2681-706.

Codina, R., Schafer, U. and Oñate, E. (1994), "Mould filling simulation using finite elements", Int. J. Num. Meth. Heat Fluid Flow, Vol. 4 No. 3, pp. 291-310.

Divone, L.V. (1999), "US industrial energy efficiency research, including a focus on metal casting", NATO/CCMS Pilot Study. Clean Products and Processes (Phase I), No. 238 pp. 13-15.

Gaston, L., Kamaraan, A. and Bellet, M. (2000), “An arbitrary Lagrangian-Eulerian finite element approach to non-steady state turbulent fluid flow with application to mould filling in casting", Int. J. Num. Meth. Fluids, Vol. 34, pp. 341-69.

Hirt, C.W. and Barkhudarov, M.R. (1998), "Lost foam casting simulation with defect prediction", in Thomas, B.G. and Beckermann, C. (Eds), Modeling of Casting, Welding and Advanced Solidification Process VIII, The Minerals, Metals \& Materials Society, San Diego, CA, pp. 51-7.

Houzeaux, G. and Codina, R. (2004), "A finite element model for the simulation of lost foam casting”, Int. J. Num. Meth. Fluids, Vol. 46, pp. 203-26. 
Hughes, T.J.R. (1995), "Multiscale phenomena: Green's functions, the Dirichlet-to-Neumann formulation, subgrid scale models, bubbles and the origins of stabilized methods", Comp. Meth. Appl. Mech. Eng., Vol. 127, pp. 387-401.

Idelsohn, S.R., Storti, M.A. and Oñate, E. (2001), "Lagrangian formulations to solve free surface incompressible inviscid fluid flows", Comp. Meth. Appl. Mech. Eng., Vol. 191, pp. 583-93.

Kuo, J-H., Chen, J-C., Pan, Y-N. and Hwang, W-S. (2003), "Mould filling analysis in lost foam casting process aluminum alloys and its experimental validation", Mater. Trans., Vol. 44, pp. 2169-74.

Lewis, R.W. and Ravindran, K. (2000), "Finite element simulation of metal casting", Int. J. Num. Meth. Eng., Vol. 47, pp. 29-59.

Lewis, R.W., Navti, S.E. and Taylor, C. (1997), "A mixed Lagrangian-Eulerian approach to modelling fluid flow during mould filling”, Int. J. Num. Meth. Fluids, Vol. 25, pp. 931-52.

Liu, X., Ramsay, C.W. and Askeland, D.R. (1994), "Study on mould filling control mechanisms in the epc process. afs 94-148", paper presented at the 98th AFS Casting Congress, Hamilton, Ontario, May, pp. 903-14.

Liu, Y., Bakhtiyarov, S.I. and Overfelt, R.A. (2002), "Numerical modeling and experimental verification of mould filling and evolved gas pressure in lost foam casting process", J. Mater. Sci., Vol. 37, pp. 2997-3003.

Mirbagheri, S.M.H., Varahram, N. and Davami, P. (2003), "3D computer simulation of melt flow and heat transfer in the lost foam casting process", Int. J. Num. Meth. Eng., Vol. 58, pp. $723-48$.

Muttin, F., Coupez, T., Bellet, M. and Chenot, J.L. (1993), "Lagrangian finite-element analysis of time-dependent viscous free-surface flow using an automatic remeshing technique application to metal casting flow", Int. J. Num. Meth. Eng., Vol. 36, pp. 2001-15.

Osher, S. and Fedkiw, R. (2002), Level Set Methods and Dynamic Implicit Surfaces, Springer, New York, NY.

Piwonka, T.S. (1990), “A comparison of lost pattern casting processes”, Mater. Design, Vol. 11 No. 6, pp. 283-90.

Radovitzky, R. and Ortiz, M. (1998), "Lagrangian finite element analysis of Newtonian fluid flows”, Int. J. Num. Meth. Eng., Vol. 43, pp. 607-19.

Wang, C., Ramsay, C.W. and Askeland, D.R. (1994), "Effect of processing parameters on mould filling for gray iron epc casting using statistical experimental techniques. afs 94-151", paper presented at the 98th AFS Casting Congress, Hamilton, Ontario, May, pp. 921-30.

Yao, X. and Shivkumar, S. (1995), "Mould filling and solidification in the lost foam process. afs 95-116”, paper presented at the 99th AFS Casting Congress, Kansas City, Missouri, April, pp. 761-5.

\section{Corresponding author}

Guillaume Houzeaux can be contacted at: guillaume.houzeaux@bsc.es

\footnotetext{
To purchase reprints of this article please e-mail: reprints@emeraldinsight.com
} Or visit our web site for further details: www.emeraldinsight.com/reprints 